\title{
Case Files of the Medical Toxicology Fellowship Training Program at the Children's Hospital of Philadelphia: A Pediatric Exploratory Sulfonylurea Ingestion
}

\author{
Diane P. Calello, $M D^{\mathrm{a}}$, Andrea Kelly, MD ${ }^{\mathrm{b}}$, Kevin C. Osterhoudt, MD, MSCE a \\ a Section of Clinical Toxicology and the Poison Control Center, Division of Emergency Medicine, The Children's Hospital \\ of Philadelphia, Philadelphia, PA \\ b Division of Endocrinology, The Children's Hospital of Philadelphia, Philadelphia, PA
}

\section{CASE PRESENTATION}

A 26-month-old boy was taken to the Emergency Department (ED) with the concern that he had ingested his mother's oral hypoglycemic medication. He and his 4-year-old sister had been caught playing with their mother's pill bottles, and the girl admitted to giving him one pill to eat. Although the implicated pills could be positively identified as extended-release glipizide (10 mg), a pill count was deemed unreliable because the medicine had been transferred from its original childproof container. In addition, too many pills were present had the mother been compliant with her prescribed dosing. The boy was developmentally normal, in good health and had no medication allergies. Additional medications reportedly available within the home included several over-the-counter preparations, prescription pain medications, and a different bottle of anti-diabetic agents. Several months earlier he had received care in another ED for an exploratory cold-medication ingestion.

The boy arrived at the ED approximately 80 minutes after the presumed exposure. He was alert, playful, and interactive, with a heart rate of 102 beats/minute and a blood pressure of $100 / 62 \mathrm{mmHg}$, and was not diaphoretic. His physical examination was unremarkable, and he was given $25 \mathrm{~g}$ of activated charcoal, in an aqueous solution flavored with cherry syrup, to drink. He was estimated to have cooperated in drinking a little more than half of his charcoal.

\section{Are Exploratory Ingestions of Sulfonylurea-Class Oral Hypoglycemic Agents Potentially Dangerous to Young Children?}

The acute mechanism of action of sulfonylurea drugs is to stimulate insulin release from pancreatic cells (Figure 1). Therefore, diabetic and non-diabetic individuals are at risk for hypoglycemia after ingesting these agents. With the high prevalence of Type II diabetes mellitus, childhood exploratory exposures to anti-diabetic medications are a common occurrence. In 2004, 4148 human sulfonylurea exposures were reported to the American Association of Poison Control Centers (AAPCC), with 1486 of those exposures occurring among children less than 6 years of age [1]. Although relevant adult mortality is recorded every year, reports of pediatric deaths from sulfonylurea poisoning are only sporadically found within Poison Center logs [2]. The low mortality rate for pediatric sulfonylurea exposures reported to Poison Centers may have several reasonable explanations: a) children, reported as exposed, may not have actually ingested the pill of concern, b) biases inherent in the AAPCC's database may lead to an underestimation of poisoning deaths, and c) in most instances, hypoglycemia is a readily identifiable and treatable condition [3]. Thus, despite a low reported incidence of sulfonylurea-induced pediatric morbidity and mortality, we believe it is imprudent to conclude that the sulfonylurea drug class is not dangerous to children.

Several review articles, including two focusing specifically on sulfonylurea drugs, have promoted sulfonylureas as a class of medications that can be highly dangerous to young children ingesting even a single dosage form [4-8]. This assertion has been

Keywords: octreotide, sulfonylurea compounds

Corresponding Author: Kevin C. Osterhoudt MD, The Poison Control Center, The Children's Hospital of Philadelphia, 34th Street \& Civic Center Boulevard, Philadelphia, PA 19104-4399. Email: OsterhoudtK@email.chop.edu 


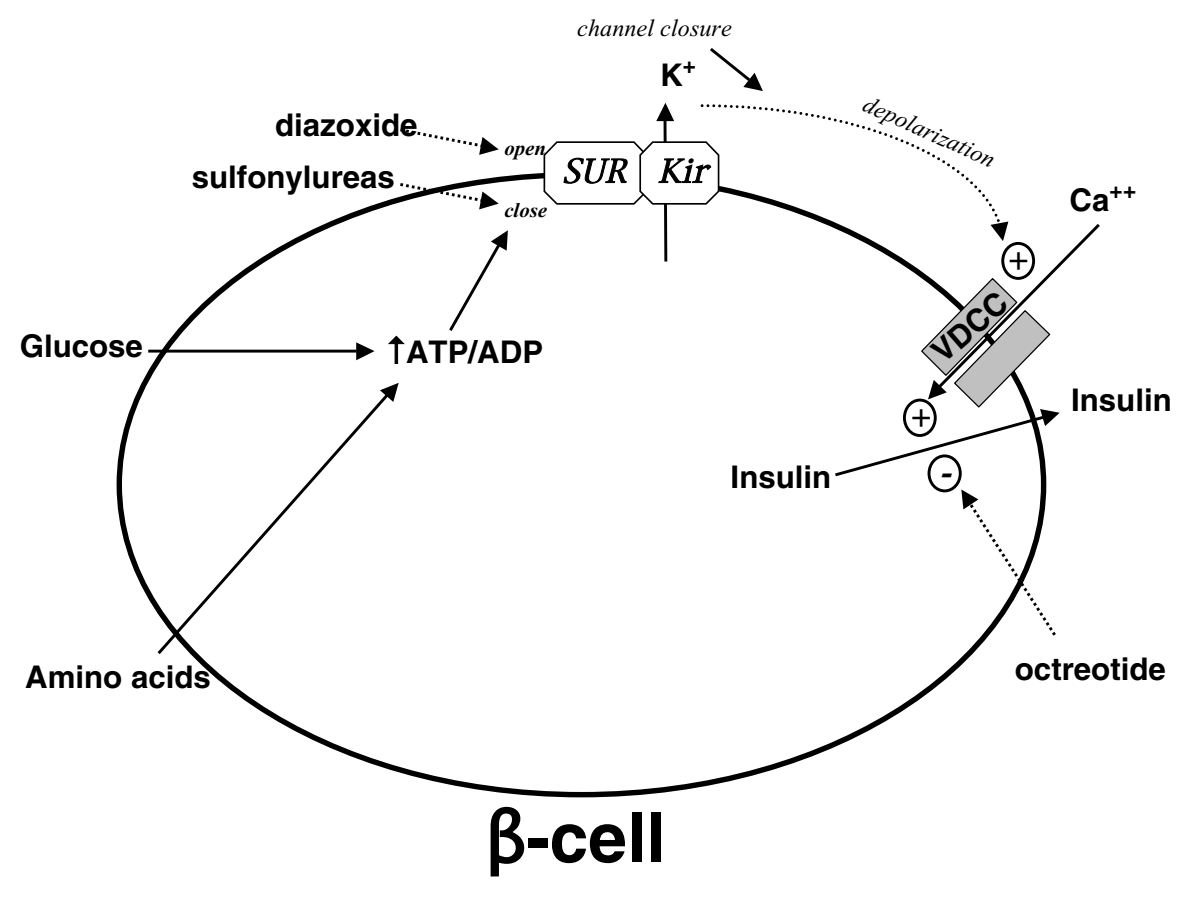

Figure 1. Insulin Secretion Pathways. In the pancreatic $\beta$-cell, metabolism of glucose and amino acids increases the adenosine triphosphate/adenosine diphosphate (ATP/ADP) ratio, leading to closure of the ATP-dependent potassium channel ( $\left.K_{A T P}\right)$ composed of the sulfonylurea receptor (SUR1) and the potassium ion pore (Kir). Closure of the $K_{\text {ATP }}$ channel causes cell membrane depolarization and calcium influx through voltage-dependent calcium channels (VDCC). This calcium influx triggers release of insulin from stored granules. Sulfonylureas, such as glipizide, promote $K_{A T P}$ channel closure and insulin release. Diazoxide, a $K_{\text {ATP }}$ agonist, acts to open the channel and inhibit insulin release. Octreotide works downstream of the VDCC to inhibit insulin release. Adapted, with permission, form: Kelly A, Stanley CA. Disorders of glutamate metabolism. Ment Retard Dev Disabili Res Rev 2001; 7: $287-295$.

reached on the basis of information published in case reports and series describing hypoglycemic morbidity from single doses of chlorpropamide $(250 \mathrm{mg})$, glipizide $(5 \mathrm{mg})$, or glyburide $(2.5 \mathrm{mg})[4,9,10,11,12]$. Offering further validation to these observations, our medical toxicology service recently consulted on two non-diabetic adults who each developed profound hypoglycemia from a single dose of a sulfonylurea drug by way of therapeutic misadventure; one of the adult patients died. Hence, we believe any pediatric sulfonylurea exposure should be considered potentially harmful.

\section{How Long After Ingestion of a Sulfonylurea-Class Oral Anti-Diabetic Medication Might a Child be Expected to Develop Hypoglycemia?}

The epidemiology of exploratory drug ingestions by young children contrasts sharply with the epidemiology of adult poisoning. Although thirty-one percent of sulfonylurea exposures reported to the AAPCC in 2004 resulted in clinical poisoning, this published data is not differentiated by age or intention [1]. The majority of children evaluated for potential sulfonylurea exposure will remain well. Therefore, it is important to determine the duration of observation necessary to appropriately categorize those poisoned from those non-poisoned.

The pharmacokinetics of most sulfonylurea drugs suggest that the peak clinical effect will be noted within 6 hours of ingestion; however, the pharmacodynamics of these drugs vary, based upon individual differences in caloric intake and glycogen stores. Drugs with a delayed peak effect, such as sustained-release glipizide, and drugs with a long duration of action, such as glyburide or chlorpropamide, might present the greatest risk for delayed onset of hypoglycemia. In one case report, a 3-year-old child ingested chlorpropamide and, although appearing well for an entire day, the child developed symptomatic hypoglycemia $(28 \mathrm{mg} / \mathrm{dl}) 36$ hours after ingestion [13]. In a case series of 40 sulfonylurea exposures, glyburide-induced hypoglycemia was discovered as late as 21 hours after ingestion, and chlorpropamideinduced hypoglycemia was discovered as late as 48 hours after ingestion [14]. Another report describes a 23-month-old boy who appeared well 5 hours after ingesting a single $5 \mathrm{mg}$ glyburide pill, but who developed symptomatic hypoglycemia several hours later during his normal sleep cycle (having received no intravenous dextrose) [4]. 
Administration of "prophylactic" intravenous dextrose may complicate the early detection of hypoglycemia after sulfonylurea ingestion. In this setting, "prophylactic" means that the patient is given intravenous dextrose (in an attempt to ward off subsequent hypoglycemia) prior to developing clinical or laboratory evidence of hypoglycemia. A retrospective case series of 93 sulfonylurea exposures in children from 1 to 16 years of age, derived from five years of experience at our Poison Center, noted that 4 of 25 children with detected hypoglycemia were discovered more than six hours after ingestion [10]. Each of the children with delayed detection of hypoglycemia had been given prophylactic dextrose. In a subsequent prospectively collected case series, three of 56 hypoglycemic children were discovered more than eight hours after ingestion, but one had received prophylactic dextrose and the other two did not have blood sugar concentrations measured within the first 12 hours [15]. Five more children with delayed discovery of sulfonylurea-induced hypoglycemia are detailed in two other reports, and only one of the five had not received prophylactic dextrose $[11,12]$.

Most children developing hypoglycemia after sulfonylurea ingestion seem to do so within 8 hours of ingestion. However, the pharmacokinetics of the drug, and the nutritional state and food intake of the patient must be considered in clinical decision making. Hypoglycemia may be delayed in some children, but the incidence of "delayed" hypoglycemia among fasting children remains unknown. We discourage the use of prophylactic intravenous dextrose because there is a theoretical risk that it will mask hypoglycemia and confound detection of the sulfonylureapoisoned child.

\section{What Are the Disposition Options for a Well-Appearing Child Brought to Medical Attention Shortly after Presumed Ingestion of a Sulfonylurea Medication?}

Untreated hypoglycemia may lead to lethargy, seizures, coma, encephalopathy, and even death. Yet, as previously stated, the vast majority of young children "exposed" to sulfonylurea drugs will show no untoward effects, and those in whom hypoglycemia is detected may be easily treated. This has led some to propose that it is appropriate to manage asymptomatic pediatric sulfonylurea exposures at home as long as they may be carefully observed by conscientious, informed caregivers with ready access and transportation to medical services [16,17]. Among all sulfonylurea exposures reported to the AAPCC in 2004, 35\% of cases were handled without referral to a health care facility, but this population may be highly represented by adult patients concerned about possible therapeutic errors [1]. The percentage of pediatric exploratory ingestions managed at home was not reported.

A more conservative approach promotes observing all pediatric sulfonylurea ingestions in a medical setting for at least eight hours [18]. Advocates of this approach suggest that children "should have free access to food," but that no prophylactic intravenous dextrose should be administered [19]. Children with no laboratory or clinical hypoglycemia within the eight hour time period are considered candidates for disposition to home. We believe that children may be able to compensate for a hyperinsulinemic state through feeding, and we have the most confidence in the sensitivity of the eight hour observation period when modified to include a period of fasting. Additionally, we have particular concerns about the vulnerability of children to hypoglycemia during the overnight fast.

In a study of fasting, in preoperative children aged four years and less, a significant risk of hypoglycemia was noted during the overnight period [20]. Among healthy children, a physiologic nadir in serum blood glucose concentration occurs at 8:00 am [21]. From these observations, it may be reasonable to suspect that children sleeping overnight may be at greatest risk of developing hypoglycemia after sulfonylurea ingestion. The overnight hours are also a time when parental supervision can be expected to be least vigilant. A prospective, descriptive series of suspected sulfonylurea ingestions tried to evaluate this theoretical concern that has been observed anecdotally, but we believe biases inherent in their data set did not allow the derivation of a valid conclusion $[15,4]$.

Since the true incidence of delayed hypoglycemia after sulfonylurea ingestion has not been precisely defined, and with concern that vulnerability to hypoglycemia is highest during the overnight fast, our Poison Center has advocated that consideration be given to the overnight medical observation of toddlers thought to have ingested adult doses of chlorpropamide, glyburide, or glipizide (especially sustained-release forms) [4]. Our Center's guidelines for the medical observation of suspected pediatric sulfonylurea ingestions are detailed in Table 1. As multiple fingerstick blood samplings are painful and stressful to young children, these guidelines also aspire to minimize such intervention. None of the presented approaches to clinical sulfonylurea management has been scientifically validated, or subjected to cost-benefit analysis.

\section{CASE CONTINUATION}

A venous blood glucose concentration one hour after arrival to the ED was $88 \mathrm{mg} / \mathrm{dl}$. Approximately 6 hours after ingestion the boy appeared lethargic, and at that time his blood glucose was found to be $55 \mathrm{mg} / \mathrm{dl}$. Following the intravenous administration of $5 \mathrm{cc} / \mathrm{kg}$ of $10 \%$ dextrose in water, the boy's affect improved and his blood glucose concentration rose to $160 \mathrm{mg} / \mathrm{dl}$.

\section{How Should a Hypoglycemic Child Suffering Sulfonylurea Poisoning be Treated?}

Recognizing hypoglycemia is the critical step to safely managing sulfonylurea ingestion. The ready availability of point-of-care glucose testing has made confirmation of hypoglycemia rapid and accessible even though commercial meters may not be calibrated to provide good discrimination at low blood glucose concentrations. Blood glucose testing is a surrogate marker for brain glucose availability, and any child with symptomatic hypoglycemia (Table 2) or a blood glucose concentration less than 
TABLE 1. The "Philadelphia Approach"* to the Asymptomatic Toddler after Sulfonylurea Ingestion

A. Children are observed in a medical setting through an overnight sleep cycle (and for a minimum period of 8 hours).

B. No prophylactic intravenous dextrose is given.

C. Patients are permitted to eat and drink normally but are subjected to an overnight fast as is typical for age.

D. Awake and alert children are not subjected to frequent, routine blood glucose testing, but an initial 3- to 6-hour post-ingestion blood glucose determination is obtained with the placement of a blood-drawing intravenous catheter.

E. Any children exhibiting symptoms of hypoglycemia have immediate blood glucose testing.

F. Sleeping children are monitored at least hourly for clinical signs such as tachycardia, diaphoresis, or agitation.** When concern arises, sleeping children are wakened. If concern persists, blood glucose concentration is measured.

G. Consideration is given to regular interval measurements of blood glucose during sleep, perhaps as frequently as every 2-3 hours depending on the patient and the medical setting (preferably via a blood-drawing intravenous line to avoid physical duress to the child).

H. Any borderline blood glucose concentrations are repeated in 1 hour.

I. If no hypoglycemic events are noted and morning, pre-breakfast, blood glucose concentrations are normal, the patients may be considered candidates for parental observation in the home.

* Derived from a consensus panel of toxicology consultants to the Poison Control Center, Philadelphia, PA

** Note that $\beta$-adrenergic antagonists, or other possible co-ingestants, may blunt the sympathetic response to hypoglycemia. Adapted, with permission, from Osterhoudt KC. Toxic topic: This treat is not so sweet: Exploratory sulfonylurea ingestion by a toddler. Pediatr Case Rev 2003; 3: 215-217.

$60 \mathrm{mg} / \mathrm{dl}$ should be treated. In the setting of sulfonylurea exposure, it is our own practice to consider blood glucose concentrations less than $70 \mathrm{mg} / \mathrm{dL}$ as "abnormal." Enteral administration of sugar is the most physiological method to provide glucose, but severely symptomatic children merit intravenous dextrose supplementation. We recommend that the emergency resuscitation of a young child with profound sulfonylurea-induced hypoglycemia begin with the rapid intravenous administration of 0.2 to $0.5 \mathrm{~g}$ of dextrose per $\mathrm{kg}$ body weight. The "rule of 50 " whereby the product of the dextrose concentration (e.g., 10\%) and the dose in cc/kg (e.g., $5 \mathrm{cc} / \mathrm{kg}$ ) equals 50 -is a popular mnemonic for calculating an intravenous dextrose dose $(0.5 \mathrm{~g} / \mathrm{kg})$ for pediatric resuscitation (Table 3). However, a lower resuscitative dose may be less prone to stimulating an exaggerated insulin response. Although exploratory sulfonylurea poisoning is

TABLE 2. Typical Symptoms and Signs of Hypoglycemia in Children*

Hunger
Irritability
Lethargy/Somnolence/Coma
Tremulousness
Tachycardia
Diaphoresis
Seizure
* Please note that the adrenergic signs of hypoglycemia may be masked when
accompanied by clinical effects of beta-adrenergic antagonists or other coingestants.

extremely rare before the age of 9 months, some clinicians eschew $50 \%$ dextrose in water in favor of $10 \%$ dextrose in water in order to minimize potential complications associated with use of hyperosmolar infusions in young children.

When continuing initial resuscitative intravenous dextrose therapy, we prefer to administer intravenous dextrose (typically, $10-15 \%$ dextrose with rate titrated to effect) by way of continuous intravenous infusion rather than via intermittent bolus dosing. A continuous infusion can be weaned in a manner that can potentially avoid the peaks and valleys of glycemic control sometimes associated with intermittent bolus dosing. Additionally, there is theoretical concern that high blood glucose concentrations associated with bolus administration of dextrose may lead to an exaggerated, counter-productive release of additional insulin during sulfonylurea poisoning.

In cases of severe sulfonylurea poisoning, hypoglycemia may prove refractory to simple enteral and intravenous dextrose administration. In such cases, additional antidotal therapies may be beneficial. Some authors have offered that glucagon, which does not inhibit insulin release, might be detrimental to the sulfonylurea-poisoned child, and they consider the drug contraindicated in this setting [22]. The counter-regulatory hormone glucagon raises blood glucose by stimulating glycogenolysis. As an insulin antagonist, glucagon should be initially effective in the setting of a sulfonylurea ingestion unless the affected child has had prolonged fasting and has not received intravenous (IV) dextrose to replenish glycogen stores. Following an IV or intramuscular bolus, blood glucose rises within minutes and peaks within 30 minutes. However, the half-life of glucagon is less than 20 minutes, and the glycemic response to glucagon abates within an hour. Nausea, vomiting, and, most importantly, rebound 


\section{TABLE 3. The "Rule of 50" for Providing $0.5 \mathrm{~g}$ of Dextrose Intravenously in the Treatment of Young Children with Sulfonylurea-Induced Hypoglycemia}

\begin{tabular}{lc}
\hline $\begin{array}{l}\text { Dextrose Concentration } \\
\text { of Intravenous Fluid }\end{array}$ & $\begin{array}{r}\text { Volume to be } \\
\text { Administered }\end{array}$ \\
\hline $10 \%$ & $5 \mathrm{cc} / \mathrm{kg}$ \\
\hline $25 \%$ & $2 \mathrm{cc} / \mathrm{kg}$ \\
\hline $50 \%$ & $1 \mathrm{cc} / \mathrm{kg}$ \\
\hline
\end{tabular}

hypoglycemia are potential problems following a single bolus dose of glucagon; and in the setting of a sulfonylurea ingestion, multiple doses may be required. An alternative to repeated glucagon boluses is continuous glucagon ( $1 \mathrm{mg}$ IV over 24 hours). This formulation is a standard therapy used from days to weeks in children awaiting pancreatectomy for congenital hyperinsulinism, another illness characterized by hypersecretion of insulin.

In theory, the ideal antidote to sulfonylurea poisoning would inhibit insulin secretion. For decades, the antihypertensive agent diazoxide has been a useful adjunct in hyperinsulinemic states. By opening ATP-sensitive potassium $\left(\mathrm{K}_{\mathrm{ATP}}\right)$ channels within pancreatic $\beta$-cells (Figure 1), diazoxide inhibits insulin release. Diazoxide is routinely administered orally $(5-15 \mathrm{mg} / \mathrm{kg} /$ day) for chronic treatment of hypoglycemia due to congenital hyperinsulinism. In the treatment of sulfonylurea ingestions in which hypoglycemia is transient, diazoxide can be a troublesome antidote, as potential side effects include tachycardia, vomiting, sodium retention, and hypotension. Diazoxide-induced hypotension is often mitigated by avoiding intravenous bolus administration. Appropriate dosing of diazoxide for sulfonylurea ingestion has not been defined by clinical trial, but a titrated continuous intravenous infusion rate of 0.1 to $2.0 \mathrm{mg} / \mathrm{kg} / \mathrm{hr}$ has been advocated [23].

The long-acting somatostatin agonist, octreotide, is now the favored antidotal adjunct for dextrose-refractory hypoglycemia due to sulfonylurea poisoning. Octreotide inhibits cyclic AMP production and calcium influx through the voltage gated calcium channel, resulting in diminished insulin secretion (Figure 1). In a human volunteer study of simulated sulfonylurea poisoning, octreotide a) reduced the requirement for intravenous dextrose compared to controls, and b) was superior to diazoxide in this regard [24]. Case series of antidotal octreotide therapy for sulfonylurea poisonings among adults have suggested benefit without adverse effects $[25,26]$.

The Children's Hospital of Philadelphia is a center for the treatment of pediatric hypoglycemic disorders and has a wealth of experience using octreotide to treat infants and children with congenital hyperinsulinism due to genetic mutations in the $\mathrm{K}_{\mathrm{ATP}}$ channel. Doses of $5-20 \mathrm{mcg} / \mathrm{kg} /$ day delivered by intermittent subcutaneous injection every 6-8 hours or by continuous intravenous or subcutaneous infusion are commonly employed. If euglycemia is not obtained at the lowest dose, the dose is incre- mentally increased by $2.5 \mathrm{mcg} / \mathrm{kg} / \mathrm{day}$. Hyperglycemia is often seen with initial dosing, but then tachyphylaxis frequently occurs over a period of a few days thus necessitating increased dosing. This phenomenon is unlikely to be an issue in the setting of a sulfonylurea ingestion in which hypoglycemia is expected to resolve within one to three days. Acute side effects of octreotide include diarrhea and abdominal discomfort [27]. Prolonged use of octreotide can cause gallstones and transient growth impairment from suppression of growth hormone secretion $[27,28,29]$. Pulmonary hypotension was identified in two pre-term infants with enterocutaneous fistulas treated with octreotide at doses of $2 \mathrm{mcg} / \mathrm{kg} /$ day and $72 \mathrm{mcg} / \mathrm{kg} /$ day over a period of 7-14 days [30]. However, the role of octreotide in this vascular manifestation remains to be established.

Despite our confidence in using octreotide to treat congenital hyperinsulinism and other disease states, limited published experience pertaining to the use of octreotide for sulfonylurea poisoning in young children exists. As such, the appropriate dose, dosing frequency, and side effect profile of octreotide in this setting has not been rigorously defined. A five-year-old boy with hypoglycemic seizures following the ingestion of glipizide was treated with lorazepam, intravenous dextrose, and octreotide [31]. Octreotide was also satisfactorily used in the treatment of a 16month-old child with refractory hypoglycemia after glyburide ingestion [32]. More recently, our medical toxicology fellowshiptraining program reported a chart review of 5 pediatric patients treated with octreotide for sulfonylurea poisoning [33]. Four of the five children experienced recurrence of hypoglycemia between 6 and 17 hours after the first octreotide dose. This anecdotal experience suggests to us that $1 \mathrm{mcg} / \mathrm{kg}$, administered intravenously or subcutaneously, is a reasonable starting dose, but that doses may need to be titrated upwards or repeated after 6 hours if hypoglycemia worsens or if sulfonylurea effect is expected to be prolonged. Occasionally, severely poisoned children may benefit from a constant intravenous infusion of octreotide titrated upward from $15 \mathrm{ng} / \mathrm{kg} / \mathrm{min}$ [19]. As episodes of hypoglycemia can recur for up to 82 hours, we also recommend that children be monitored after cessation of intravenous dextrose therapy and receive no more octreotide for at least 8 hours, and then be fasted safely through an overnight sleep cycle, before discharge [14]. In our case series, one patient (while euglycemic) developed a brief period of apnea and hypertension, of unclear etiology, 30 minutes following his intravenous octreotide dose.

\section{CASE CONCLUSION}

Despite several bolus doses of $25 \%$ dextrose in water, and the infusion of $15 \%$ dextrose in water at a rate exceeding one and one-half times the rate for maintenance fluid requirements, hypoglycemia was recurrent and persistent. Octreotide, at a dose of $1.5 \mathrm{mcg} / \mathrm{kg}$, was administered intravenously, and no subsequent hypoglycemic episodes were noted. The dextrose infusion was quickly weaned to a $10 \%$ solution at maintenance fluid requirements, and was discontinued altogether at 
approximately 11 hours after the octreotide dose. The child later slept overnight with a 6:00 am fasting blood glucose concentration of $78 \mathrm{mg} / \mathrm{dl}$. The family was provided with poisoning prevention guidance, and the boy was discharged home. The family was called by the Poison Center two days after hospital discharge, at which time the family reported that the boy continued to appear well.

\section{REFERENCES}

1. Watson WA, Litovitz TL, Rodgers GC, Klein-Schwartz W, Reid N, Youniss J, et al. 2004 annual report of the American association of poison control centers toxic exposure surveillance system. Am J Emerg Med 2005; 23: 589-666.

2. Litovitz TL, Klein-Schwartz W, Dyer KS, Shannon M, Lee S, Powers M. 1997 annual report of the American Association of Poison Control Centers toxic exposure surveillance system. Am J Emerg Med 1998; 16: 443-497.

3. Osterhoudt KC. Principles of epidemiology and research design. In: Goldfrank LR, Flomenbaum NE, Lewin NA, Howland MA, Hoffman RS, Nelson LS, editors. Goldfrank's Toxicologic Emergencies (7th ed.). New York: McGraw Hill; 2002. pp 1789-1797.

4. Osterhoudt KC. Toxic topic: This treat is not so sweet: exploratory sulfonylurea ingestion by a toddler. Pediatr Case Reviews 2003; 4: 215-217.

5. Little GL, Boniface KS. Are one or two dangerous? Sulfonylurea exposure in toddlers. J Emerg Med 2005; 28: 305-310.

6. Osterhoudt KC. The toxic toddler: drugs that can kill in small doses. Contemp Pediatr 2000; 17: 73-88.

7. Bar-Oz B. Levichek Z. Koren G. Medications that can be fatal for a toddler with one tablet or teaspoonful: a 2004 update. Paediatric Drugs 2004; 6:123-6.

8. Michael JB, Sztajnkrycer MD. Deadly pediatric poisons: nine common agents that kill at low doses. Emerg Med Clin NA 2004; 22: 1019-1050.

9. Sketris L, Wheeler D. York S. Hypoglycemic coma induced by inadvertent administration of glyburide. Drug Intell Clin Pharm 1984; 18: 142-143.

10. Quadrani DA, Spiller HA, Widder P. Five-year retrospective evaluation of sulfonylurea ingestion in children. J Toxicol Clin Toxicol 1996; 34: 267-270.

11. Borowski H, Caraccio T, Mofensen H. Sulfonylurea ingestion in children: is an 8-hour observation period sufficient? J Pediatr 1998; 133: 584.

12. Szlatenyi CS, Capes KF, Wang RY. Delayed hypoglycemia in a child after ingestion of a single glipizide tablet. Ann Emerg Med 1998; 31: 773-776.

13. Greenberg B, Weihl C, HugG. Chlorpropamide poisoning. Pediatrics 1968; 41: 145-147.

14. Palatnick W, Meatheral RC, Tenenbein M. Clinical spectrum of sulfonylurea overdose and experience with diazoxide therapy. Arch Intern Med 1991; 151: 1859-1862.
15. Spiller HA, Villalobos D, Krenzolak EP, Anderson BD, Gorman SE, Rose SR, et al. Prospective multi-center study of sulfonylurea ingestion in children. J Pediatr 1997; 131: 141-146.

16. Robertson WO. Sulfonylurea ingestions: hospitalization not mandatory. J Toxicol Clin Toxicol 1997; 35: 115-116.

17. Robertson WO. Delayed hypoglycemia after ingestion of a single glipizide tablet [letter, comment]. Ann Emerg Med 1999; 33: 130.

18. Burkhart KK. When does hypoglycemia develop after sulfonylurea ingestion? Ann Emerg Med 1998; 31: 771-772.

19. Spiller HA. Management of sulfonylurea ingestions. Pediatr Emerg Care 1999; 15: 227-230.

20. Thomas DK. Hypoglycemia in children before operation: its incidence and prevention. Br J Anesth 1974; 46:66-68.

21. Marin G, Rose SR, Kibarian M, Barnes K, Cassorla F. Absence of dawn phenomenon in normal children and adolescents. Diabetes Care 1988; 11:393-396.

22. Graudins A, Linden CH, Ferm RP. Diagnosis and treatment of sulfonylurea-induced hyperinsulinemic hypoglycemia. Am J Emerg Med 1997; 15: 95-96.

23. Ingels M. Hypoglycemic agents and insulin. In: Erickson TB, Ahrens WR, Aks SE, Baum CR, Ling LJ, editors. Pediatric Toxicology. New York: McGraw Hill; 2005. pp 277-282.

24. Boyle PJ, Justice K, Krentz AJ, Nagy RJ, Schade DS. Octreotide reverses hyperinsulinemia and prevents hypoglycemia induced by sulfonylurea overdoses. J Clin Endocrinol Metab 1993; 76: 752-756.

25. McLaughlin SA, Crandall CS, McKinney PE. Octreotide: an antidote for sulfonylurea-induced hypoglycemia. Ann Emerg Med 2000; 36: 133-138.

26. Carr R, Zed PJ. Octreotide for sulfonylurea-induced hypoglycemia following overdose. Annals Pharmacother 2002; 36: 1727-1732.

27. Glaser B, Hirsch HJ, Landau H. Persistent hyperinsulinemic hypoglycemia of infancy: long-term octreotide treatment without pancreatectomy. J Pediatr. 1993 Oct;123(4):644-50.

28. Radetti G, Gentili L, Paganini C, Messner H. Cholelithiasis in a newborn following treatment with the somatostatin analogue octreotide. Eur J Pediatr. 2000 Jul;159(7):550.

29. Thornton PS, Alter CA, Katz LE, Baker L, Stanley CA Short- and long-term use of octreotide in the treatment of congenital hyperinsulinism. J Pediatr. 1993 Oct;123(4):637-43.

30. Arevalo RP, Bullabh P, Krauss AN, Auld PA, Spigland N. Octreotide-induced hypoxemia and pulmonary hypertension in premature neonates. J Pediatr Surg. 2003 Feb;38(2):251-3.

31. Mordel A, Sivilotti MLA, Old AC, Ferm RP. Octreotide for pediatric sulfonylurea poisoning [abstract]. J Toxicol Clin Toxicol 1998; 36: 437.

32. Kent DA, Main BA, Friesen MS. Use of octreotide in sulfonylurea poisoning in a child [abstract]. J Toxicol Clin Toxicol 1993; $41: 669$.

33. Calello DP, Osterhoudt KC, Henretig FM, Perrone J. Octreotide for pediatric sulfonylurea overdose: Review of 5 cases [abstract]. Clin Toxicol 2005; 43: 671. 